\title{
Estudio de la temperatura de cristalización, en la obtención de vitrocerámicos de muy bajo coeficiente de expansión térmica a partir de mineral espodumeno de origen nacional y óxido de titanio como agente de nucleación.
}

\section{Study of the crystallization temperature in obtaining glass- ceramics with a very low coefficient of thermal expansion from spodumene mineral of national origin and titanium oxide as nucleating agent.}

Presentación: 6-7/10/2020

\section{Doctorando:}

\section{Marisa Adriana Sierra}

Grupo de investigación y Desarrollo en Tecnologías Químicas Aplicadas (IDETQA), Facultad Regional Buenos Aires, Universidad Tecnológica Nacional - Argentina perezia2004@yahoo.com.ar

\section{Director:}

\section{Ulises Gilabert}

\section{Resumen}

Los vitrocerámicos con base en aluminosilicatos de Litio han adquirido relevancia debido a su bajo coeficiente de expansión térmica (CET), además de brindar buena resistencia mecánica, maquinabilidad y buen desempeño frente al ataque químico. Estas características les otorgan diversidad de aplicaciones, paneles de hornos, cocinas eléctricas, aplicaciones en óptica, como así también, componentes internos de válvulas y accesorios de la industria de procesos químicos que deben asegurar la estanqueidad de los equipos, aun cuando son sometidos a choques térmicos significativos. En este trabajo se desarrollaron vitrocerámicos a diferentes temperaturas de cristalización, utilizando Espodumeno mineral $\left(\mathrm{LiAlSi}_{2} \mathrm{O}_{6}\right)$ de un yacimiento argentino, dado el interés que tiene para nuestro país agregarle valor a sus recursos minerales de litio.

La obtención de vitrocerámicos a partir del mineral, tiene como ventajas los menores costos, si se los compara con los devenidos por el uso de reactivos de calidad analítica, y la valoración del recurso nacional. Además nos interesa determinar la acción nucleante del óxido de Titanio.

Palabras clave: Vitrocerámicos, coeficiente de expansión térmica lineal, Sellos, nucleantes.

\footnotetext{
Abstract

Glass- ceramics based on Lithium aluminosilicate have acquired relevance due to their low coefficient of thermal expansion (CET), in addition to providing good mechanical resistance and good performance against chemical attack. These characteristics give them a diversity of applications, oven panels, electric cookers, optical applications, as well as internal components of valves and accessories in the chemical process industry that must ensure the tightness of the equipment even when subjected to significant thermal shocks. In this work, glass-ceramics were developed at different crystallization temperatures, using mineral Spodumene ( $\left(\mathrm{LiAlSi}_{2} \mathrm{O}_{6}\right)$ from an Argentine deposit given the interest that our country has to add value to its lithium mineral resources.
} 
Obtaining glass-ceramics from mineral has the advantages of lower costs when compared to those incurred by the use of analytical quality reagents and the valuation of the national mining resource. We are also interested in determining the nucleating action of Titanium oxides.

Keywords: Glass- Ceramics, coefficient of thermal expansion, seal, nucleating agent.

\section{Introducción}

Los aluminosilicatos de litio se han usado en la industria del vidrio y la cerámica como fundentes y como cargas de bajo coeficiente de expansión en cerámica blanca (R. P. Hevia; C. Gil; J. B. Carda, 2006). En los tempranos 1960 una patente de Stookey señaló la eficiencia del $\mathrm{TiO}_{2}$ como agente nucleante y se iniciaron una serie de estudios sobre el sistema $\mathrm{Li}_{2} \mathrm{O}-\mathrm{Al}_{2} \mathrm{O}_{3}-\mathrm{SiO}_{2}$ (LAS) y las aplicaciones de los vitrocerámicos derivados (S.D. Stookey, 1960). .

$\mathrm{La}$ familia de los aluminosilicatos de litio incluye compuestos con la fórmula LiAlSixO $2 \mathrm{X}+2$. Cationes como $\mathrm{Mg}^{2+} \mathrm{y}$ $\mathrm{Zn}^{2+}$ pueden sustituir al $\mathrm{Li}^{1+}$ y x puede variar entre 2 y 6 . También el $\mathrm{AlPO}_{4}$ puede reemplazar parcialmente a $\mathrm{SiO}_{2}$. Además el Aluminio puede ser sustituido por cationes con número de oxidación $+20+3$.

Los vitrocerámicos de LAS tienen como principal atractivo sus bajos coeficientes de expansión, buena resistencia química y buenas propiedades mecánicas (D.U. Tulyaganov; S. Agathopoulos; H.R. Fernandez, 2004) [5].Las mejores propiedades térmicas se obtienen por la generación de cristales de soluciones sólidas de aluminosilicatos de litio, cuarzo de alta $\mathrm{T}$, keatita (sílice tetragonal, Beta-eucriptita $\left(\mathrm{LiAlSiO}_{4}\right)$, Beta-espodumeno $\left(\mathrm{LiAl}\left(\mathrm{SiO}_{3}\right)_{2}\right)$, insertos dentro de la matriz vítrea. Se pueden usar en partes de precisión de sistemas ópticos, paneles de cocinas, ventanas de hornos, fuentes para hornos y las partes internas de válvulas y accesorios sometidos a saltos térmicos y erosión (H. Bach; Dieter Krause, 2005).

Las variaciones dimensionales de piezas sometidas a choque térmico, suelen generar grandes inconvenientes de filtraciones, perdidas y fugas en los componentes internos de válvulas y de accesorios de la industria de procesos químicos que deben asegurar resistencia al desgaste y estanqueidad.

La fabricación de un vitrocerámicos del tipo LAS, implica obtener en primer lugar un vidrio de base para luego conformar algún objeto. Sigue un tratamiento térmico que convierte al vidrio en un vitrocerámico. Para este tipo de vitrocerámicos se suele incluir $\mathrm{TiO}_{2}$ y/o $\mathrm{ZrO}_{2}$ como agentes de nucleación (D. Ovono; P. Pradeau; S. Berre; G. Bruno, 2013).

La producción de un vitrocerámico LAS es relativamente costosa pues son costosas las materias primas de grado analítico requeridas y por la elevada temperatura necesaria para obtener los vidrios de base $\left(>1600{ }^{\circ} \mathrm{C}\right)$. Como alternativa se trabaja con un mineral con el contenido de aluminosilicatos de litio, como el espodumeno que permitirá bajar costo dando relevancia al recurso natural.

\section{Desarrollo}

Como materias primas para obtener los vidrios de base se utilizó mineral espodumeno de origen nacional, muestra del El Alto, Catamarca, cuya composición química se determinó en un trabajo previo, mediante fluorescencia de rayos $\mathrm{X}$ y espectrometría de absorción atómica determinando la composición química en porcentajes en masa es: $\mathrm{SiO}_{2}$ (72,14\%), $\mathrm{Al}_{2} \mathrm{O}_{3}$ (20,43\%), $\mathrm{Li}_{2} \mathrm{O}$ (5,18\%), $\mathrm{Fe}_{2} \mathrm{O}_{3}(0,94), \mathrm{Na}_{2} \mathrm{O}$ (0,94\%), $\mathrm{K}_{2} \mathrm{O}(0,28 \%), \mathrm{CaO}(0,08 \%), \mathrm{MgO}(0,04), \mathrm{TiO}_{2}$ $(0,01 \%)$ ( R. P. Hevia; C. Gil; J. B. Carda, 2006). y, por el otro, ácido bórico y dióxido de titanio, ambos de grado analítico. Tabla 1.

\begin{tabular}{|c|c|}
\hline Componente & Porcentaje en masa \\
\hline Espodumeno & 91,06 \\
\hline Oxido de Boro & 5,15 \\
\hline Oxido de Titanio & 3,79 \\
\hline
\end{tabular}

Tabla 1: Composición del vidrio de base

Todos los componentes del vidrio de base se mezclaron intensamente en un mortero antes de llevarlos al horno sometiéndolos al siguiente ciclo térmico: Temperatura ambiente hasta los $1600^{\circ} \mathrm{C}$ a una velocidad de $10^{\circ} \mathrm{C} / \mathrm{minuto} \mathrm{y}$ permaneció a dicha temperatura durante 1 hora. Se realizó la frita y luego se sometió a molienda y tamizado hasta lograr un tamaño de partícula pasante por malla ASTM 100 y retenido en malla ASTM 200. Posteriormente se 
caracterizó el vidrio por calorimetría diferencial de barrido (CDB) (equipo: TA-Q600), grafica 1 y dilatometría mecánica (equipo: NETZSCH 402-C), grafica 2, este último se realizó para tener la referencia del vidrio.

Sobre los resultados del análisis térmico diferencial se seleccionaron las temperaturas para los ciclos térmicos correspondientes al tratamiento térmico para obtener así cuatro vitrocerámicos diferentes. Tabla 2. Para dichos tratamientos se realizaron probetas prismáticas que fueron aglutinadas y prensadas previamente al tratamiento térmico.

Sobre cada uno de los vitrocerámicos obtenidos se obtuvieron las respectivas curvas dilatométricas, evaluadas entre temperatura ambiente y $300^{\circ} \mathrm{C}$, se incluyeron las determinaciones del vidrio de base y del vidrio patrón de silicio. Grafica 2. El registro de los datos se encuentra en la tabla 3.

Posteriormente para identificar la fase cristalina los vitrocerámicos y el vidrio base fueron nuevamente reducidas a polvo y caracterizadas por DRX, (Philips X'Pert MPD). Grafica 3. Además se registraron micrografías, por medio del SEM, de todos los vitrocerámicos y del vidrio de base para poder establecer el tamaño de partícula de la fase cristalina identificada. Fig1.

\begin{tabular}{|l|c|c|c|c|}
\hline & $\begin{array}{c}\text { Temperatura } \\
\text { de nucleación. } \\
{ }^{\circ} \mathbf{C}\end{array}$ & $\begin{array}{c}\text { Tiempo del } \\
\text { tratamiento. } \\
\text { horas }\end{array}$ & $\begin{array}{c}\text { Temperatura } \\
\text { de } \\
\text { cristalización. } \\
{ }^{\circ} \mathbf{C}\end{array}$ & $\begin{array}{c}\text { Tiempo del } \\
\text { tratamiento. } \\
\text { horas }\end{array}$ \\
\hline VC1 & 670 & 2 & 780 & 2 \\
\hline VC2 & 670 & 2 & 850 & 2 \\
\hline VC3 & 670 & 2 & 900 & 2 \\
\hline CV4 & 670 & 2 & 1000 & 2 \\
\hline
\end{tabular}

Tabla 2: Tratamientos térmicos aplicados en la obtención de vitrocerámicos.

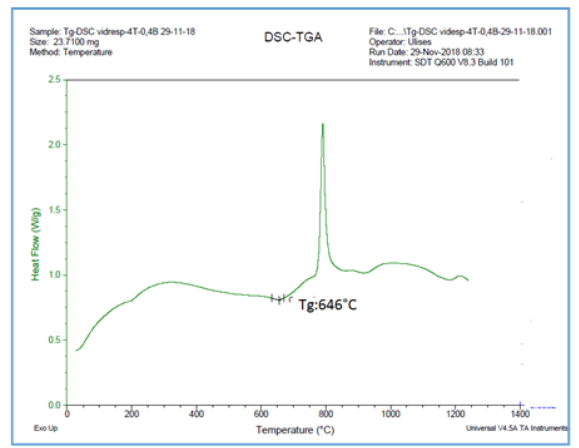

Gráfica 1: CDB del vidrio de base. TA-Q600

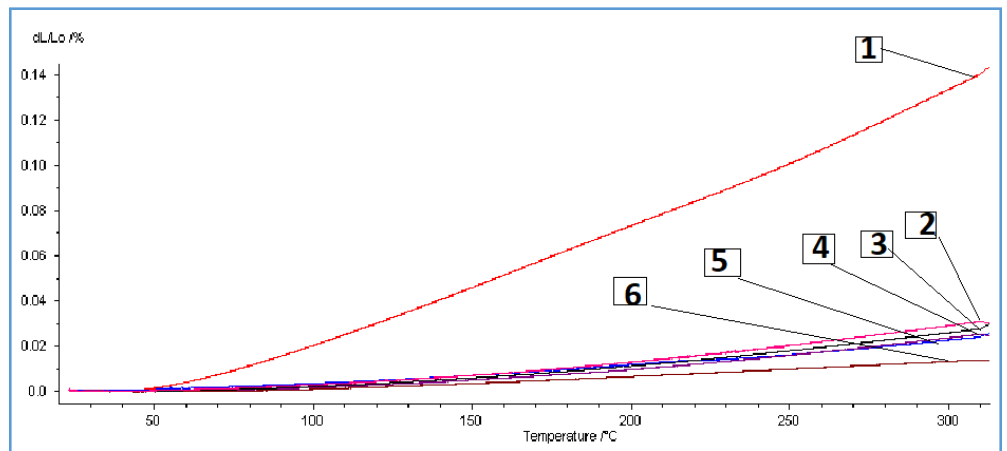

Gráfica 2: Curvas dilatométricas: (1) Vesp. (Vidrio de base), (2) VC4, (3) VC2, (4) VC1, (5) VC3, (6) Vidrio de $\mathrm{SiO}_{2}$. 


\begin{tabular}{|c|c|c|c|c|c|c|}
\hline $1 /{ }^{\circ} \mathrm{C}$ & $\begin{array}{c}\text { Vidrio } \\
\text { Patón } \\
\text { de } \\
\text { silicio } \\
\end{array}$ & $\begin{array}{l}\text { Vidrio } \\
\text { Esp. }\end{array}$ & $\begin{array}{c}\text { VC1 } \\
\text { TC:780 } \\
C\end{array}$ & $\begin{array}{c}\text { VC2 } \\
\text { TC: } 850^{\circ} \mathrm{C}\end{array}$ & $\begin{array}{c}\text { VC3 } \\
\text { Tc: } 900^{\circ} \mathrm{C}\end{array}$ & $\begin{array}{c}\text { VC4 } \\
\text { TC: } 1000^{\circ} \mathrm{C}\end{array}$ \\
\hline $\begin{array}{c}\text { CETL } \\
\left(30^{\circ} \mathrm{C}-\right. \\
\left.300^{\circ} \mathrm{C}\right)\end{array}$ & $\begin{array}{c}0,54 \\
E-6\end{array}$ & $\begin{array}{c}5,27 \mathrm{E}- \\
6\end{array}$ & 0,79 E-6 & 0,88 E-6 & 0,75 E-6 & $1,01 \mathrm{E}-6$ \\
\hline
\end{tabular}

Tabla 3: Registro de los coeficientes de expansión térmica Media Intervalo de $0^{\circ} \mathrm{C}$ a $300^{\circ} \mathrm{C}$.
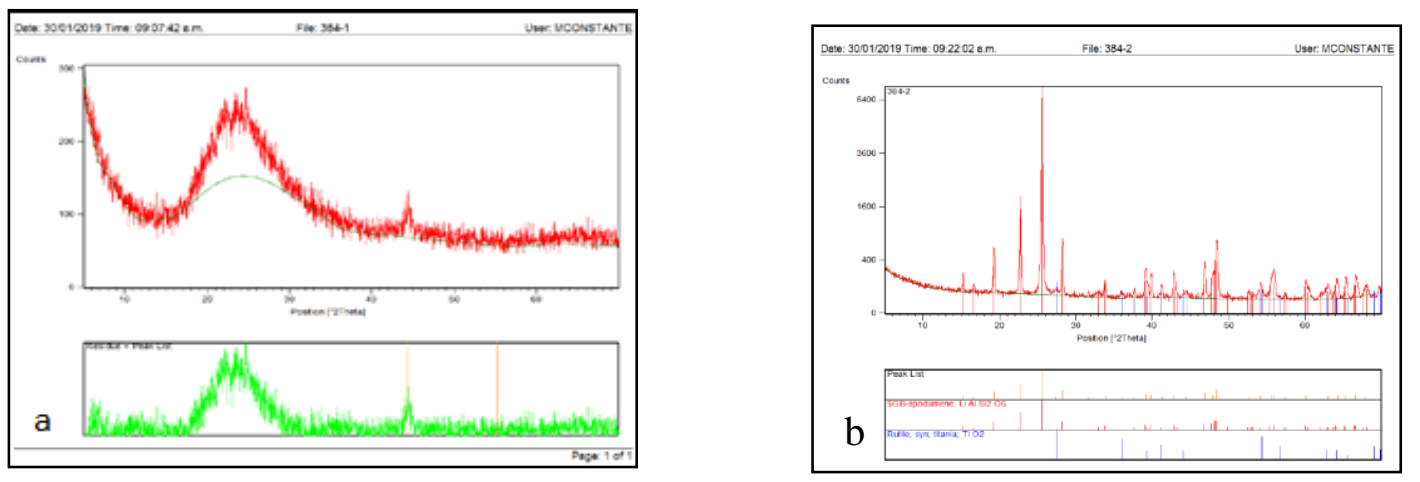

Gráfica 3: a: Difractograma del vidrio de base y b: del vitrocerámico CV1. (Philips X'Pert MPD).

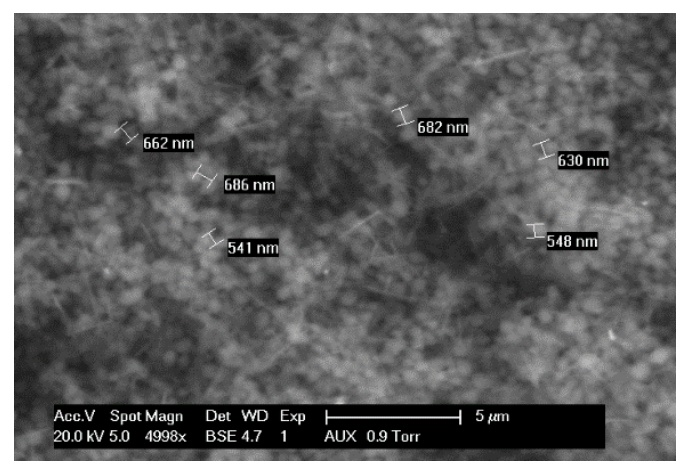

Figura 1: SEM CV3

\section{Resultados}

Sobre los resultados del ensayo de $\mathrm{CDB}$, donde se observa $\mathrm{Tg}=646^{\circ} \mathrm{C}$ y Temperatura del pico máximo, Tpmax $=790^{\circ} \mathrm{C}$, se seleccionaron las temperaturas del tratamiento térmico realizado sobre las probetas. Los primeros resultados mostrados en la gráfica 2 , son alentadores, se han obtenido vitrocerámicos de muy bajo CET, con una importante diferencia respecto del vidrio de base. Esta diferencia nos indica una transformación en la estructura, de vítrea a una fase cristalina confirmada en el estudio de DRX, donde aparece identificada la fase del ß- espodumeno, conjuntamente con el rutilo como componente minoritario, correspondiente con el agente de nucleación, Grafica 3. Cuando se comparan los resultados con el vidrio de origen, no hay dudas de que el CET sufrió una notable disminución ya que en todos los casos sus valores son cercanos al del patrón de vidrio de sílice. Los bajos CET de los vitrocerámicos obtenidos se deben a la formación de cristales de $\beta$-espodumeno como consecuencia de los tratamientos térmicos, dentro de las probetas obtenidas, es más notable, el del vitrocerámico cristalizado a $900{ }^{\circ} \mathrm{C}$ (VC3) y con un valor muy cercano, el cristalizado a $780^{\circ} \mathrm{C}(\mathrm{VC} 1)$.

En todas las probetas se identificó $\beta$-espodumeno, por los ensayos de DRX (Philips X'Pert MPD) y de las micrografías realizadas (SEM) figura 1, en particular el VC3 presenta tamaños de cristales de distribución homogénea, esféricos, aislados, dentro de la matriz vítrea uniforme, con diámetros de partícula entre 500 y $600 \mathrm{~nm}$, a lo que atribuimos el mejor resultado en los CET en el intervalo $30-300^{\circ} \mathrm{C}\left(0,75 \cdot 10-6^{\circ} \mathrm{C}-1\right)$. Se visualizaron también en forma eventual algunos cristales aciculares que no fueron identificados en la DRX dada su poca cantidad. Dentro de los 
resultados es importante también destacar que el mejor resultado no está relacionado con la mayor temperatura de cristalización, dato relevante desde el punto de vista del gasto energético.

\section{Referencias}

H. Bach, Dieter Krause. (2005). Low Thermal Expansion Glass Ceramics, Springer, 3.

R. P. Hevia, C. Gil, J. B. Carda, (2006). Tratamiento Mineral de Espodumeno en la Industria. Cerámica. Caracterización y aprovechamiento tecnológico, Qualicer, 55-60.

D. Ovono Ovono, P. Pradeau, S. Berre, G. Bruno, (2013). Conditions for Crystallization of LAS GlassCeramics as a Function of Nucleating Agent Amount and Heat Treatment, International Journal of Applied Glass Science 4,1, 20-30.

S.D. Stookey, (1960). Method of making ceramics and product thereof, US Patent 2920971.

D.U. Tulyaganov, S. Agathopoulos, H.R. Fernandez, (2004). Synthesis of Lithium aluminosilicate glass and glass- ceramics from spodumene material, Ceramics international, 30, 1023-1030. 